\title{
Decrease in opioid and intra-articular corticosteroid burden after intra-articular hyaluronic acid for knee osteoarthritis treatment
}

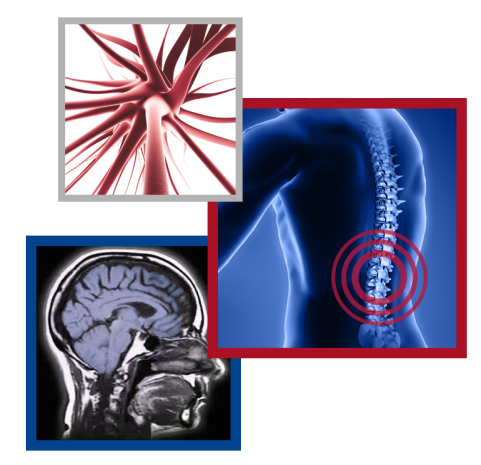

\author{
Faizan Niazi ${ }^{1}$, Kevin L Ong*,2 (D), Vasco Deon Kidd ${ }^{3}$, Edmund Lau ${ }^{4}$ Steven M Kurtz², \\ Stanley H Dysart ${ }^{5}$ \& Gerard Malanga ${ }^{6}$ \\ ${ }^{1}$ Ferring Pharmaceuticals, Inc., 100 Interpace Parkway, Parsippany, NJ 07054, USA \\ 2Exponent, Inc., 3440 Market St, Suite 600, Philadelphia, PA 19104, USA \\ ${ }^{3}$ Department of Orthopaedic Surgery, University of California Irvine (UCI Health), 101 The City Drive South, Orange, CA 92868, \\ USA \\ ${ }^{4}$ Exponent, Inc., 149 Commonwealth Ave, Menlo Park, CA 94025, USA \\ ${ }^{5}$ Pinnacle Orthopaedics \& Sports Medicine Specialists, 652 Church Street, Marietta, GA 30060, USA \\ ${ }^{6}$ New Jersey Regenerative Institute, 197 Ridgedale Ave \#210, Cedar Knolls, NJ 07927, USA; Clinical Professor Dept. of Physical \\ Medicine \& Rehabilitation, Rutgers School of Medicine - NJ Medical School Newark, NJ 07109, USA \\ *Author for correspondence: Tel.: +1 215594 8800; Fax: +1 215594 8899; kong@exponent.com
}

\begin{abstract}
Aim: We studied changes in opioid prescriptions and corticosteroid injection use for knee osteoarthritis patients before and after intra-articular hyaluronic acid (HA) use and opioid prescriptions before and after knee arthroplasty (KA). Materials \& methods: A total of 1,017,578 knee osteoarthritis members were ascertained from a commercial claims database (Health Intelligence Company LLC, IL, USA) using ICD9/ICD10 diagnosis codes. Results: Eighty two percent of HA patients did not fill opioid prescriptions postinjection, with $54 \%$ of opioid users discontinuing fills. Two-thirds of KA patients filled opioid prescriptions within 6 months postsurgery, with $78 \%$ of opioid users continuing fills and $62 \%$ of nonusers initiating use. Conclusion: Alternative therapies, such as $\mathrm{HA}$, that reduce opioid use may alleviate opioid addiction risks for KA patients who use opioids in the pre- and postoperative periods.
\end{abstract}

First draft submitted: 13 July 2020; Accepted for publication: 20 August 2020; Published online: 7 September 2020

Keywords: hyaluronic acid • intra-articular corticosteroids $\bullet$ knee arthroplasty $\bullet$ knee osteoarthritis $\bullet$ opioids

Opioid use has received extensive national attention in the US due to misuse and dependence, as well as the elevated risk of overdose and overdose-related deaths. In response to the opioid epidemic, the CDC published guidelines in 2016 on the appropriateness of prescribing opioids for chronic pain $[1,2]$. The guidelines expressed that clinicians need to carefully reassess evidence of individual benefits and risks when increasing opioid dosage to at least 50 morphine milligram equivalents (MME) per day. They also recommend avoiding or carefully justifying a decision for dosing at least $90 \mathrm{MME} /$ day. Opioids are one of the options for managing pain from knee osteoarthritis (OA), although the latest American Academy of Orthopaedic Surgeons (AAOS) Clinical Practice Guidelines (CPG) provided an inconclusive recommendation for its use [3]. Opioids appear to be used by up to about $30 \%$ of knee OA patients [4,5], but substantial statewide variation exists that are not fully explained by differences in access to healthcare providers, state policies or patient case-mix [6].

Pre-operative opioid use is linked to a variety of adverse outcomes following knee arthroplasty (KA) [7-15]. About a third to half of KA patients receive opioids 3-12 months before surgery $[7,8]$. However, pre-operative use is associated with greater risk of readmission, complications and revision surgery following total KA [9]. Pre-operative opioid users also tend to have less pain relief, poorer quality of life $[11,14]$ and are at increased risk of chronic postoperative opioid use $[10,12,13,15]$. Research has shown as many as $64 \%$ of chronic opioid users continue opioids more than 1 year postoperatively [12,15]. Moreover, $22 \%$ of opioid naive patients filled opioid prescriptions at 1 year 
postsurgery [15]. In addition, continuous opioid use after 90 days postsurgery is associated with elevated revision arthroplasty risk [16].

Alternative, nonsurgical methods for knee pain management include intra-articular injections (corticosteroids [CS] or hyaluronic acid [HA]), physical therapy, weight loss, home exercise programs and use of nonsteroidal antiinflammatory drugs. The use of HA has been reported to reduce the need for analgesic or rescue medication [17-20]. However, the AAOS CPG did not recommend HA based on a meta-analysis of its clinical efficacy, while an inconclusive recommendation was provided for CS [3]. With the ongoing concerns about the use and abuse of opioids [21] and availability of alternate means for treating pain, the present study sought to evaluate: the changes in opioid prescriptions and CS injection use patterns for knee OA patients before and after the use of HA and the changes in opioid prescriptions before and after KA. The study hypotheses were: there are significant changes in opioid prescriptions and CS injection use patterns for knee OA patients before and after the use of HA; there are significant changes in opioid prescriptions before and after KA and the changes in opioid prescription before and after the use of $\mathrm{HA}$ are not different from those before and after KA.

\section{Materials \& methods}

Newly-diagnosed members with knee OA were ascertained from a large commercial claims database (Health Intelligence Company LLC, IL, USA) containing Health Insurance Portability and Accountability Act (HIPAA) compliant de-identified data of more than 100 million patients with continuous coverage from 2012 to 2016. All medical and prescription claims, membership data and provider information were collected in the dataset; these data were linked to allow identification and tracking of all medical and prescription drug claims for a given beneficiary covered. Over-the-counter drugs are not included in this dataset. Members who had uninterrupted health insurance coverage in the health plan, in other words, continuous enrollment, for at least 6 months during study period were included. Newly-diagnosed knee OA patients were ascertained from International Classification of Diseases, Ninth and Tenth Revisions, for knee OA diagnoses or nonspecific OA with concurrent diagnosis of knee pain ICD-9 $(715.06,715.16,715.26,715.36,715.86,715.96)$ or ICD-10 M17; ICD-9 ([715.08, 715.18, 715.28, 715.38, 715.88, 715.98] and 719.46) or ICD-10 (M19.9 and M25.56); ICD-9 ([715.09, 715.19, 715.29, 715.39, 715.89, 715.99] and 719.46) or ICD-10 ([M15.0, M15.3, M15.8] and M25.56); ICD-9 ([715.00, $715.10,715.20,715.30,715.80,715.90]$ and 719.46) or ICD-10 ([M15.4, M15.9, M19.9] and M25.56). The identification of a new knee OA diagnosis was based on the first knee OA diagnosis during the data period, with at least a 6-month look-back period with no knee OA diagnosis. This was a mid-range look-back period based on prior studies $[7,18,22,23]$ that have used $0-12$ months to look-back for diagnosis of conditions and/or comorbidities. Patients were excluded if they had unknown age or gender, unknown state, enrollment gap of more than 3 months, with HA or KA before the first knee OA diagnosis or did not have pharmacy benefits. HA use was identified using Healthcare Common Procedure Coding System codes (J7321, J7323, J7324, J7325, J7326, J7327, J7328, C9471, Q9980), with concurrent diagnosis coding on the same claim to indicate likelihood of knee injection (711.x6, 712.x6, 715.x6, 716.x6, 717.x, 718.x6, 719.x6, M00.x6, M01.x6, M02.x6, M11.x6, M12.x6, M13.x6, M24.x6, M25.x6, 717, 836, 844, M17, M22, M23, S83). KA was identified using procedure codes 81.54, 27446, 27447, OSRC0J, 0SRT0J, 0SRU0J, OSRV0J, 0SRW0J, 0SRC0L, 0SRD0L, 0SRD0J (ICD-10 Procedure Coding System codes without 0 SPC0 or 0 SPD0 on the same claim). A total of 1,722,689 knee OA patients were initially identified, but 1,017,578 patients remained for analysis after excluding those without pharmacy benefit (Figure 1). Patient age, gender and census region characteristics were compiled (Table 1).

The opioid burden was estimated from opioid prescription fills (Table 2) within 7 days of a medical claim with a knee OA diagnosis. The 7-day period was used as an attempt to link opioid prescriptions with the knee OA condition by restricting them to prescription fills within a week of a medical claim with a knee OA diagnosis, but the prescription itself could be for any number of days of supply, for example, 3 months of supply. A sensitivity analysis was performed using 30 days instead of 7 days as the period after the knee OA-related claims to identify opioid prescription fills as being linked to knee OA. The 30 days were intended to indicate the period following a knee-OA medical claim during which an opioid prescription was filled and not indicative of the number of days of supply. CS injections were also identified from the knee OA-related medical claims. Intra-articular CS from knee OA-related claims using J0702, J0704 (expired after 31 December 2010), J1020, J1030, J1040, J1094, J1100, J1700, J1710, J1720, J2650, J2920, J2930, J3300, J3301, J3302, J3303 (eff 1 January 2009). Opioid users or patients who received CS injections were identified within 12 months following knee OA diagnosis and within 6 months before and after HA injection. Those who filled opioid prescriptions before and after undergoing KA were 
Table 1. Knee osteoarthritis patient characteristics.

\begin{tabular}{|c|c|c|c|c|c|c|}
\hline & & \multirow[b]{2}{*}{ Knee OA } & \multicolumn{2}{|c|}{ No HA } & \multicolumn{2}{|c|}{ HA } \\
\hline & & & KA & No KA & KA & No KA \\
\hline Sex & Age (years) & & & & & \\
\hline \multirow[t]{11}{*}{ Female } & $0-39$ & 44,763 & 327 & 39,578 & 147 & 4711 \\
\hline & $40-44$ & 39,779 & 808 & 33,853 & 393 & 4725 \\
\hline & $45-49$ & 67,130 & 2348 & 55,499 & 1228 & 8055 \\
\hline & $50-54$ & 109,064 & 5963 & 87,640 & 2651 & 12,810 \\
\hline & $55-59$ & 135,153 & 9675 & 106,674 & 3961 & 14,843 \\
\hline & $60-64$ & 128,076 & 10,383 & 101,568 & 3113 & 13,012 \\
\hline & $65-69$ & 26,646 & 2201 & 21,246 & 618 & 2581 \\
\hline & $70-74$ & 7356 & 625 & 5816 & 165 & 750 \\
\hline & $75-79$ & 3039 & 219 & 2470 & 60 & 290 \\
\hline & $80+$ & 2301 & 87 & 2008 & 22 & 184 \\
\hline & Total & 563,307 & 32,636 & 456,352 & 12,358 & 61,961 \\
\hline \multirow[t]{11}{*}{ Male } & $0-39$ & 42,142 & 231 & 38,128 & 96 & 3687 \\
\hline & $40-44$ & 34,879 & 658 & 30,213 & 301 & 3707 \\
\hline & $45-49$ & 54,916 & 1834 & 46,435 & 808 & 5839 \\
\hline & $50-54$ & 84,851 & 5094 & 69,132 & 1768 & 8857 \\
\hline & $55-59$ & 105,857 & 9014 & 83,225 & 2774 & 10,844 \\
\hline & $60-64$ & 96,653 & 9409 & 75,636 & 2347 & 9261 \\
\hline & $65-69$ & 23,581 & 2365 & 18,516 & 539 & 2161 \\
\hline & $70-74$ & 7098 & 688 & 5560 & 170 & 680 \\
\hline & $75-79$ & 2782 & 240 & 2211 & 65 & 266 \\
\hline & $80+$ & 1512 & 85 & 1258 & 16 & 153 \\
\hline & Total & 454,271 & 29,618 & 370,314 & 8884 & 45,455 \\
\hline \multicolumn{7}{|c|}{ Census region } \\
\hline \multirow[t]{2}{*}{ Midwest } & East north central & 158,510 & 9545 & 123,598 & 4403 & 20,964 \\
\hline & West north central & 91,097 & 8544 & 72,078 & 2504 & 7971 \\
\hline \multirow[t]{2}{*}{ Northeast } & Atlantic & 180,686 & 8191 & 145,722 & 3430 & 23,343 \\
\hline & New England & 67,613 & 4373 & 60,025 & 598 & 2617 \\
\hline \multirow[t]{3}{*}{ South } & East south central & 102,358 & 5395 & 82,903 & 2360 & 11,700 \\
\hline & South Atlantic & 185,428 & 10,607 & 155,308 & 3203 & 16,310 \\
\hline & West south central & 149,810 & 9230 & 120,097 & 3184 & 17,299 \\
\hline \multirow[t]{2}{*}{ West } & Mountain & 35,782 & 3442 & 27,887 & 851 & 3602 \\
\hline & Pacific & 46,294 & 2927 & 39,048 & 709 & 3610 \\
\hline
\end{tabular}

\section{Table 2. Opioids identified from the pharmacy claims.}

\section{Opioids}

Alfentanyl or alfentani

Anileridine

Alphaprodine

Bromadol

Buprenorphine

Butorphanol

Codeine

Dextropropoxyphene

Dihydroetorphine

Dihydrocodeine

Diamorphine

\begin{tabular}{ll} 
Etorphine & Oxycodone \\
\hline Fentanyl & Oxymorphone \\
\hline Heroin & Oxymorphone \\
\hline Hydrocodone & Propoxyphene \\
\hline Hydromorphone & Remifentanyl or remifentanil \\
\hline Levorphanol & Sufentanyl or sufentanil \\
\hline Meperidine/pethidine & Tramadol \\
\hline Methadose & Tapentadol \\
\hline Methadone & Hydroxymitragynine \\
\hline Morphine & \\
\hline Nalbuphine & \\
\hline
\end{tabular}




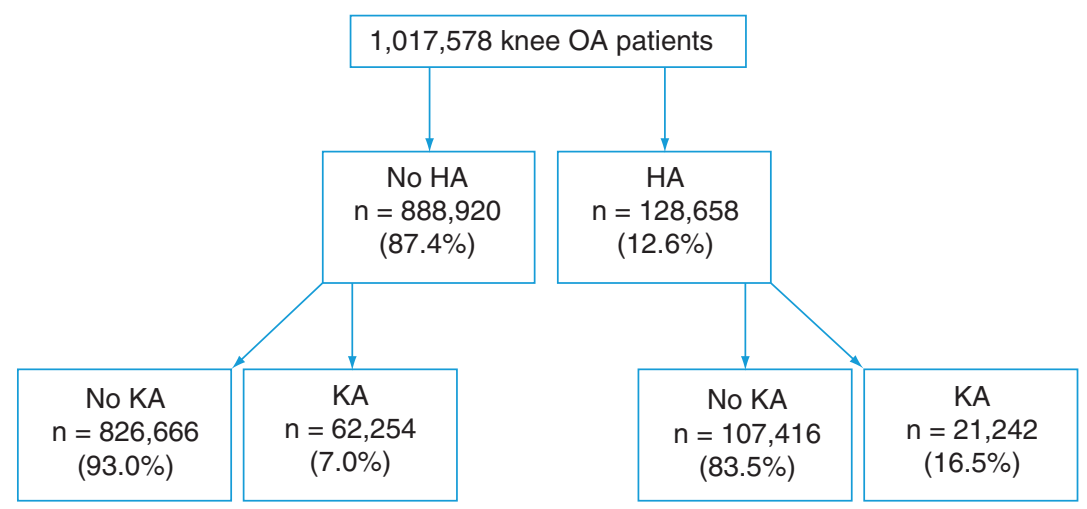

Figure 1. Knee osteoarthritis patient distribution.

HA: Hyaluronic acid; KA: Knee arthroplasty; OA: Osteoarthritis.
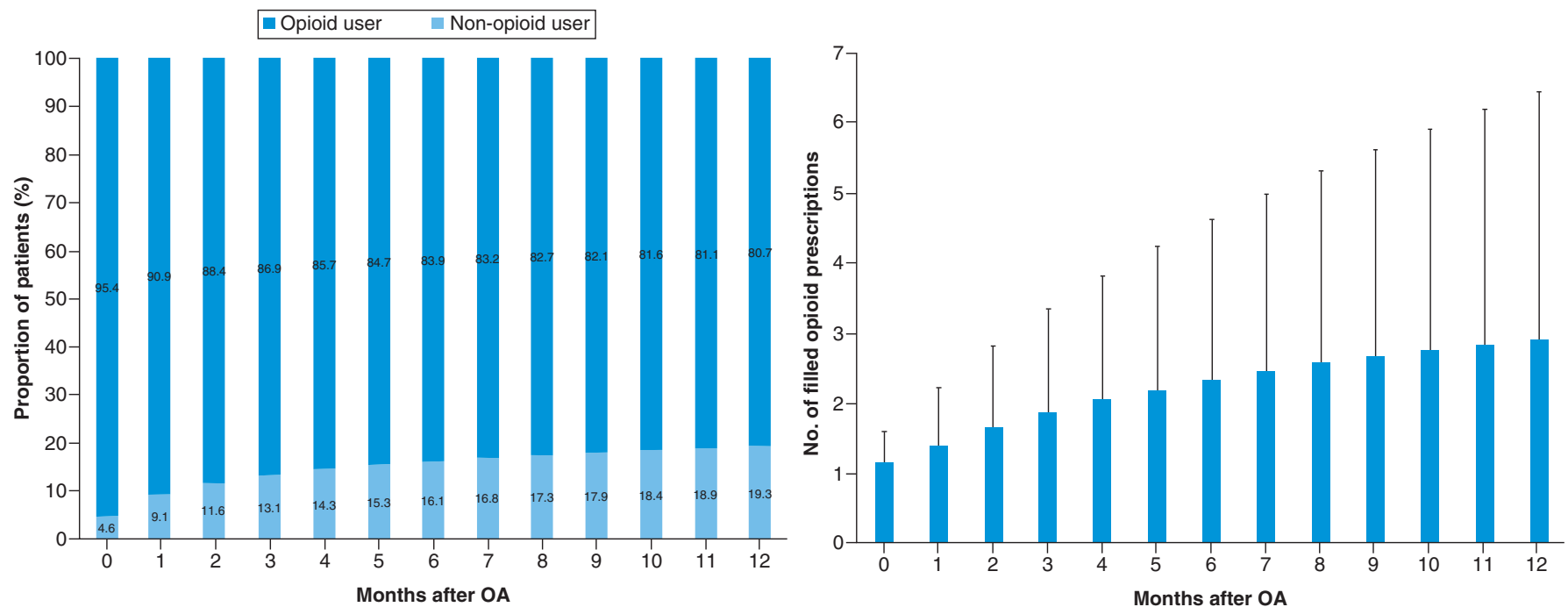

Figure 2. Opioid burden within 12 months post-knee osteoarthritis diagnosis (left: percent of patients; right: number of filled prescriptions).

OA: Osteoarthritis.

also identified. Changes in opioid prescriptions and CS injection use patterns for knee OA patients before and after the use of HA, as well as changes in opioid prescriptions before and after KA were analyzed using one-sample binomial proportion test. Changes in opioid prescription before and after the use of HA were also compared with those before and after KA using chi-square tests.

\section{Results}

A total of 1,017,578 knee OA patients were identified in this cohort (Table 1), of which $12.6 \%(\mathrm{n}=128,658)$ received at least one HA injection and 8.2\% $(\mathrm{n}=83,496)$ underwent KA. Within 6 months after knee OA diagnosis, $16.1 \%$ of patients were opioid users, with an average of $2.3 \pm 2.3$ prescription fills (Figure 2). The percent of opioid users increased to $19.3 \%$ of the patients by 12 months, with an average $2.9 \pm 3.5$ prescription fills. Almost a quarter of the knee OA patients (23.5\%) received CS injections within 1 year after knee OA diagnosis, with an average of $1.6 \pm 1.0$ injections per patient (Figure 3).

Overall, $82 \%$ of all HA patients did not fill opioid prescriptions in the 6 months after receiving the HA injection, with $75.6 \%$ as nonusers and $6.7 \%$ as discontinued opioid users (Figure 4 ). There was a $54 \%$ reduction in the number of opioid users at 6 months among those patients who had received a HA injection (Table 3). At each time 

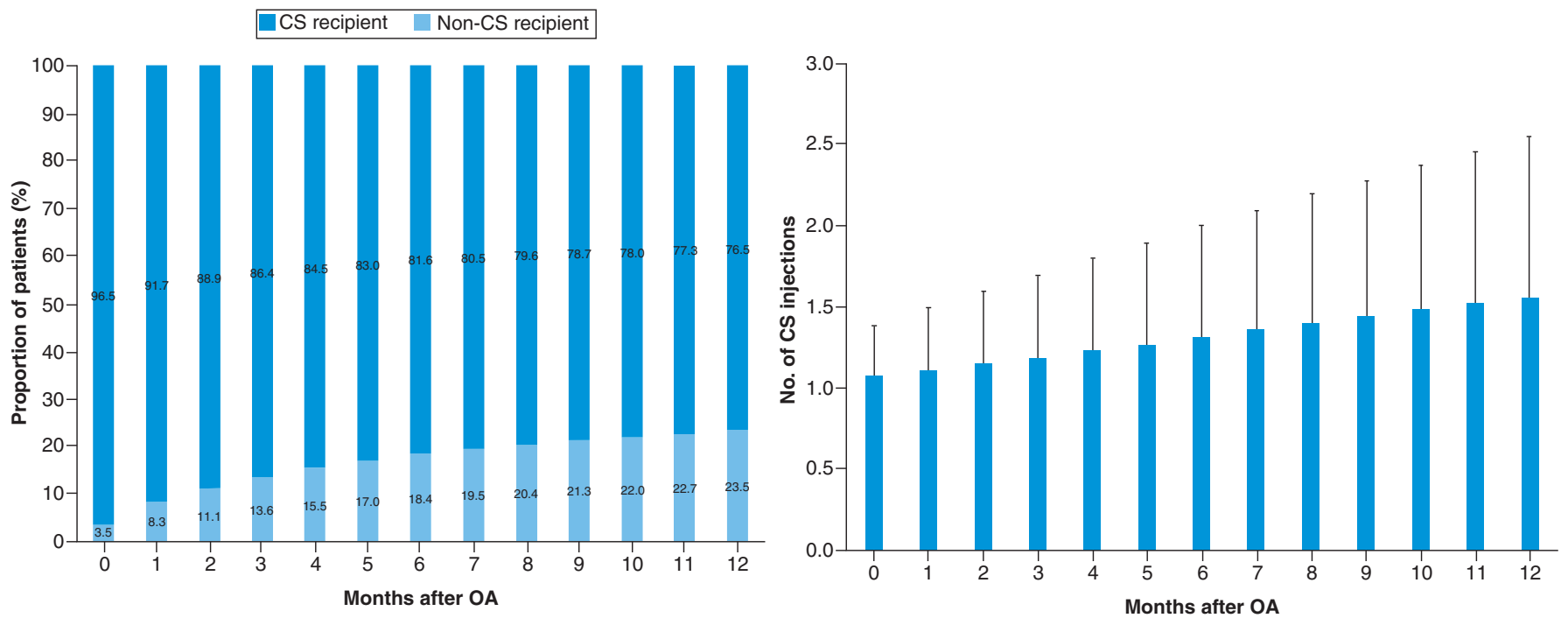

Figure 3. Corticosteroids injection burden within 12 months post-knee osteoarthritis diagnosis (left: percent of patients; right: number of injections).

CS: Corticosteroid; OA: Osteoarthritis.

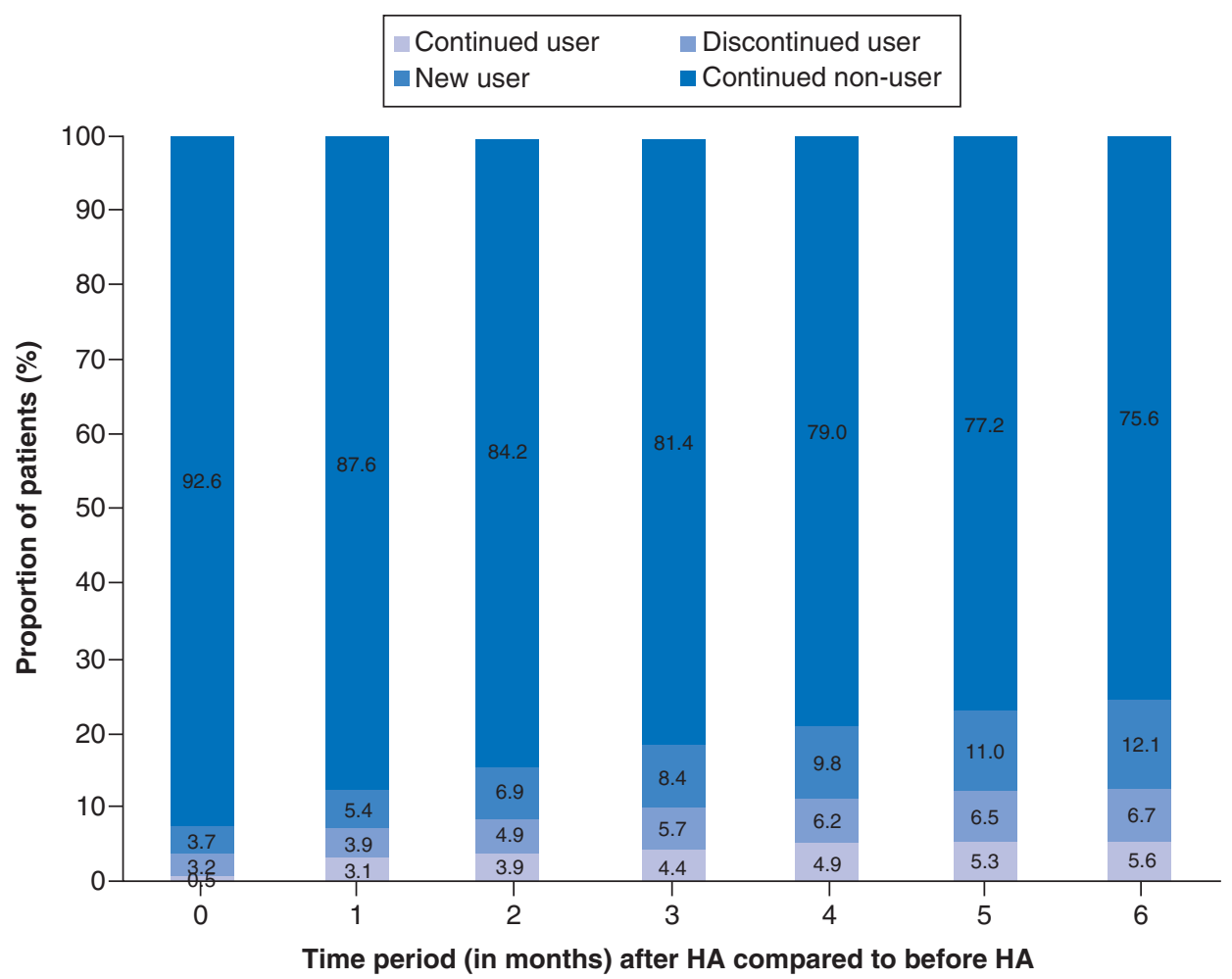

Figure 4. Opioid burden within 6 months pre- and post-hyaluronic acid use.

HA: Hyaluronic acid.

\begin{tabular}{|l|l|}
\hline Table 3. Changes in opioid burden in the 6 months before and after hyaluronic acid or knee arthroplasty. \\
\begin{tabular}{|lll|}
\hline & Percent of users who continued use & Percent of nonusers who initiated use \\
\hline After HA & 46 & 14 \\
\hline After KA & 78 & 62 \\
\hline HA: Hyaluronic acid; KA: Knee arthroplasty. & & \\
\hline
\end{tabular}
\end{tabular}




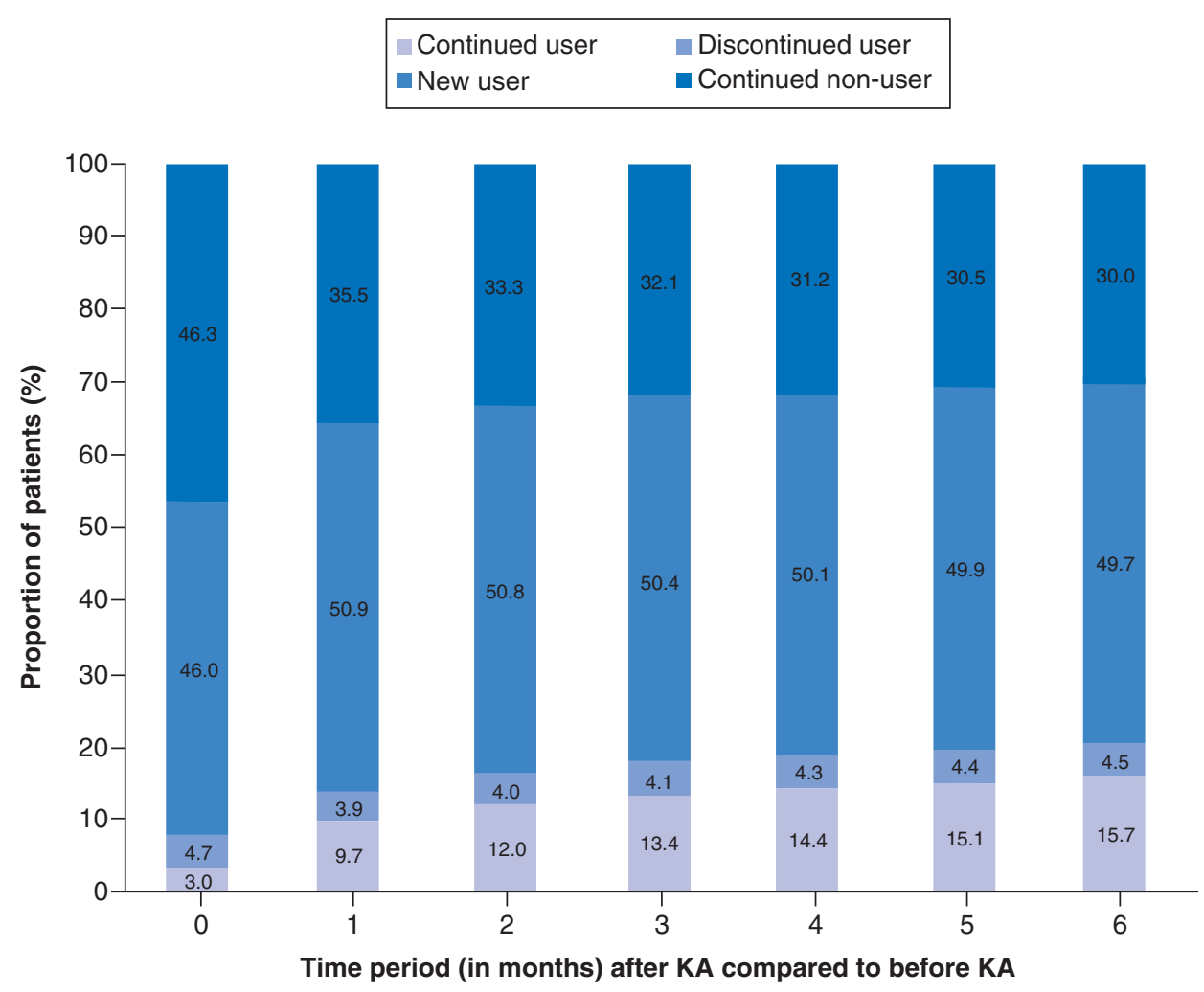

Figure 5. Opioid burden within 6 months pre- and post-knee arthroplasty. KA: Knee arthroplasty.

period, the proportion of opioid users who discontinued use after receiving the HA injection was found to be a statistically significant change $(\mathrm{p}<0.001)$. Only $14 \%$ of previous nonusers became new users in the 6 months after the HA injection ( $\mathrm{p}<0.001$ for all time periods). Of all KA patients, two-thirds filled opioid prescriptions within 3 months (63.8\%) and 6 months (65.4\%) postsurgery (Figure 5). A total of $78 \%$ of the opioid users continued to fill prescriptions in the 6 months post-KA, while $62 \%$ of nonopioid users initiated use during the same time interval (Table 3). The proportion of new opioid users and the proportion of opioid users who discontinued use after KA were both found to be a significant change for all time periods $(\mathrm{p}<0.001)$. Based on this breakdown, approximately three-quarters (76\%) of those opioid users within 6 months following KA were new users (Figure 5). When comparing the changes in opioid burden between HA patients and KA patients, the proportion of new opioid users was found to be greater for KA patients than HA patients ( $\mathrm{p}<0.001$ for all time periods). The proportion of new opioid users ranged from 3.9\% at month 0 to $13.8 \%$ at month 6 for HA patients and ranged from $49.9 \%$ at month 0 to $62.5 \%$ at month 6 for KA patients. There was a significantly greater proportion of opioid users who discontinued use for HA patients than KA patients (1.4-times greater at month 0-2.4-times greater at month $6 ; \mathrm{p}<0.001$ for all time periods).

Regarding CS use, 78\% of all HA patients did not receive an injection in the 6 months following their HA injection (Figure 6). At each time period, the proportion of HA patients who underwent a CS injection before HA, but did not receive another CS injection after HA, was found to be statistically significant $(p<0.001) .71 \%$ of the patients who underwent a CS injection did not receive another CS injection, within 6 months after receiving their HA injection. Only $16 \%$ of those who initially had not received CS injections subsequently received a CS injection within 6 months after their HA injection; this was found to be statistically significant for all time periods $(\mathrm{p}<0.001)$.

For the sensitivity analysis of extending the opioid prescription period based on pharmacy claims filled within 30 days, instead of 7 days, of any knee OA-related claims; the percent of opioid users increased from 19.3 to $24.1 \%$ within 1 year following knee OA diagnosis, with an average of $3.2 \pm 4.0$ prescription fills. In the 6 months after receiving the HA injection, $78 \%$ of all HA patients did not fill opioid prescriptions, with about half $(49 \%)$ of the 


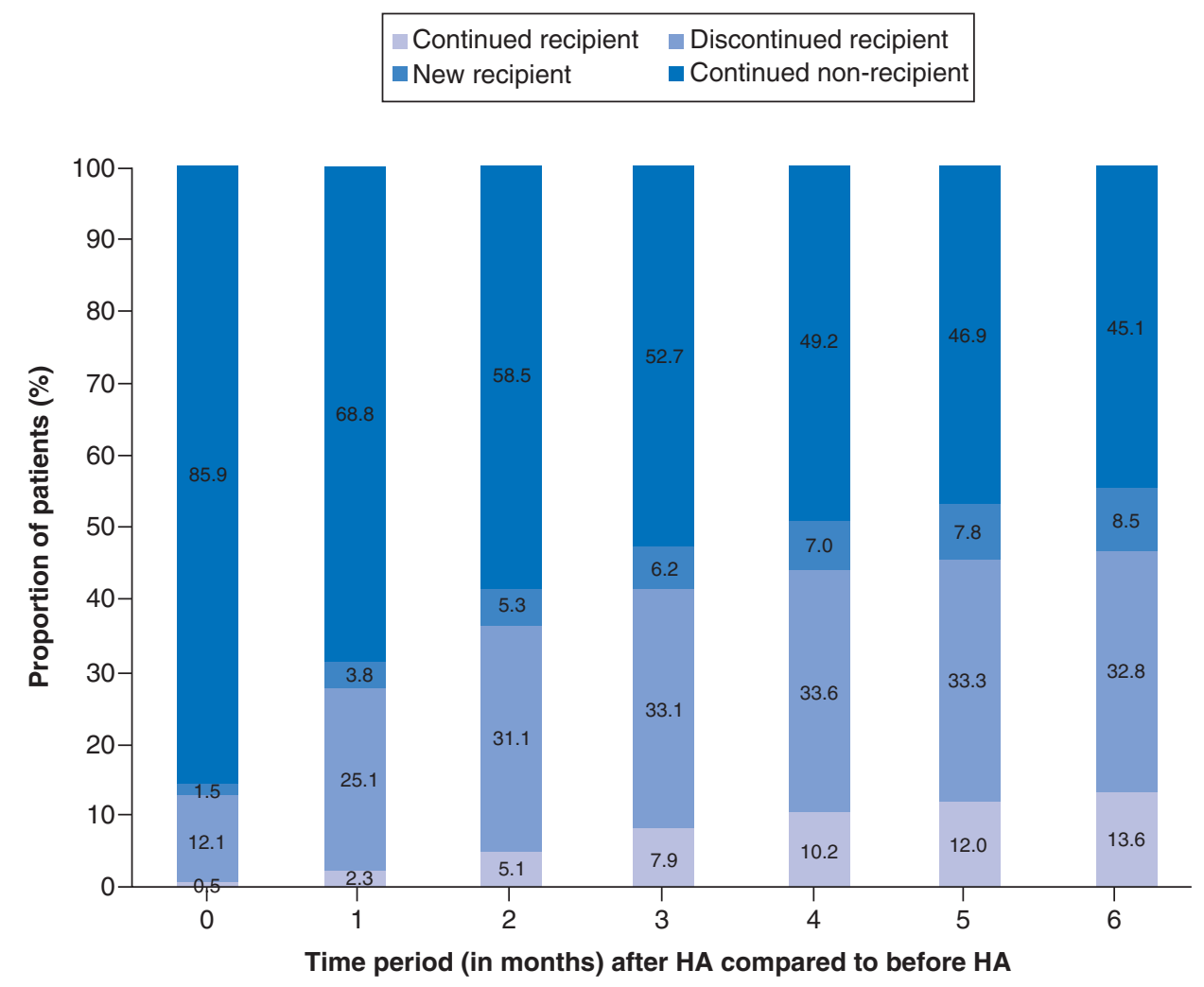

Figure 6. Corticosteroids injection burden within 6 months pre- and post-hyaluronic acid use. HA: Hyaluronic acid.

opioid users stopping use post-HA and only $17 \%$ becoming new opioid users. In contrast, only $29 \%$ of KA patients were not opioid users within 6 months postsurgery. About three-quarters (71\%) opioid users post-KA were new users. $82 \%$ of patients who received a new opioid prescription following KA continued to fill prescriptions in the 6 months post-KA. These results from the sensitivity analysis, along with conclusions drawn from the results, did not change substantially.

\section{Discussion}

Pain management is an important component of nonsurgical treatment in symptomatic knee OA and often includes the use of opioids, which are not without risks. Our study showed that about one-fifth of knee OA patients filled opioid prescriptions, along with about one-quarter who required at least one CS injection, within a year of diagnosis. Moreover, over half of the opioid users discontinued filling new prescriptions after receiving a HA injection. After receiving $\mathrm{HA}$ injections, new opioid users remained low with $14 \%$ of nonusers initiating use. Overall, $82 \%$ of HA patients did not use opioids within 6 months following the HA injection. Conversely, about two-thirds of KA patients filled opioid prescriptions 3-6 months postsurgery, with the vast majority (about 75\%) of those as a new opioid user. The proportion of new opioid users was found to be greater for KA patients than HA patients, while the proportion of opioid users who discontinued use was greater for HA patients than KA patients. Most patients receiving HA (78\%) also did not require additional CS injections in the 6 months after their HA injection. With increasing concerns surrounding the risks of opioid addiction, consideration should be given to whether alternative OA therapies, such as CS and HA injections, are effective in reducing the opioid burden.

The present study has several limitations, many of which were related to the use of administrative claims data. We relied on the diagnosis and procedure codes that were in the claims data, however, their accuracy could not be independently verified. The severity of knee OA could not be determined for these patients, hence the patients who received HA injections may be at an earlier disease stage than those who underwent KA. However, the proportion of the HA cohort who received a CS injection in the prior 6 months was not lower than that for the KA cohort (HA: $46 \%$ vs KA: $29 \%$ ). In addition, there was a greater percentage of opioid users for the KA cohort (20.2\%) than the 
HA cohort (12.3\%) before receiving their respective interventions. This does not explain the dramatic differences in nonopioid users who initiated use after their respective interventions (KA: 62\% vs HA: 14\%). The study also lacked clinically pertinent data on pain and function to correlate with opioid prescription and CS burden. There may be confounders that also explain the differences in usage before and after each of the HA and KA interventions than just the interventions themselves. However, the potential confounders may be evened out with the large patient population in both cohorts (HA: 128,658; KA: 83,496). Others have also described the association between diabetes and pain severity in those with localized OA [24], as well as with knee pain in those with knee OA [25,26]. Although we analyzed whether patients filled opioid prescriptions, the dosages and number of days supplied were not examined to calculate MME. Thus, we were unable to compare against the CDC guidelines of 50-90 MME per day $[1,2]$. However, this does not obviate the risk of being accessible to opioids with potential for abuse, addiction, overdose and death. Filled prescriptions also do not equate to consumption rates, nonetheless, self-reported opioid use has its limitations in under-reporting and validity $[27,28]$. One cannot directly link the prescriptions with knee OA, but we attempted to provide linkage by requiring a knee OA medical claim within a short time period (7 days) preceding the prescription fill and see this as a strength to the study compared with other studies that may have included all prescription fills. The duration provided a short lag for the patient to fill the prescription following their knee OA-associated claim. We examined the sensitivity of the 7-day assumption by extending it to 30 days, but the results did not change substantially. It is also unclear to what extent the prescriptions may also be associated with other etiologies, even though we used a knee OA diagnosis on the medical claim as a trigger for inclusion.

Although KA is considered by some to be the only treatment for knee OA, in the present study, two-thirds of all KA patients filled opioid prescriptions within 6 months postsurgery. This suggests that pain was still not fully managed by the surgical intervention. Although the majority of those were patients with a new opioid prescription, most $(78 \%)$ of the prior patients prescribed an opioid still continued filling prescriptions after KA. There was also a more than fourfold greater proportion of patients not previously requiring an opioid who initiated use following KA than HA treatment (KA: 62\% vs HA: 14\%). The growing opioid burden in the KA cohort can have substantial societal impact, such as risk of overdose and overdose-related deaths [1,2]. Research demonstrates that long-term pre-operative opioid use prior to KA has been associated with poor pain relief following KA [11,14], poorer health-related quality of life [11] and greater likelihood of continuing opioid use postsurgery [10,12,13,15]. Namba and coworkers reported that $60 \%$ of primary total knee replacement patients used opioids prior to surgery, but $41 \%$ of the patients still continued using opioids 3 months after surgery [12]. They also identified pre-operative opioid use as a significant risk factor for greater postoperative opioid use. Zarling et al. found that $64 \%$ of chronic opioid users before primary joint arthroplasty were still being prescribed opioids at 1 year postsurgery compared with $22 \%$ of the control group [15]. In a study of 66,950 total knee arthroplasty (TKA) patients with available opioid data in the Humana Health insurance system, 54.8\% were found to use opioids within 1 year pre-operatively [13]. About a third (34.8\%) of those opioid users continued to use opioids chronically after TKA with prescriptions for over 6 contiguous months. This study also noted that the risk of chronic postoperative opioid use was almost four-times greater for patients with pre-operative opioid use. Moreover, pre-operative opioid use can have a negative effect on the success of KA. Ben-Ari et al. determined that $39.1 \%$ of TKA patients were on long-term opioid use of greater than three consecutive months in the year prior to surgery [22]. Those patients were found to have elevated revision risk (odds ratio of 1.40; 95\% CI: 1.19-1.64) in the first postoperative year. Persistent opioid use following KA may also have negative clinical consequences. Those who required persistent opioid use beyond 90 days after KA have been associated with elevated 1-year revision risk by more than twofold compared with nonopioid users [16]. Despite the concerns with chronic opioid use, opioids received an inconclusive recommendation as a treatment option for knee OA according to the AAOS CPG [3]. It is unclear if opioid use is appropriate for knee OA patients, as well as for KA patients or which patients will develop opioid use disorder or opioid-related adverse events [29].

Although the AAOS CPG provided an inconclusive recommendation for opioids, it did not recommend HA due to a perceived lack of clinical efficacy and not to potential safety concerns [3]. Primary knee replacement surgery has its corresponding risks of complications and revision surgery, with $10-34 \%$ estimated to experience long-term pain after surgery [30], while HA has a known safety profile [31]. Furthermore, our study demonstrated a reduction in opioid burden after patients receive intra-articular HA. Not only was there a substantially lower percentage of opioid naive patients initiating use, more than half of the opioid users discontinued filling new opioid prescriptions after HA injection. Other clinical trials have also reported the reduced need for analgesic or rescue medication following HA use [17-20]. A 34.9\% reduction in acetaminophen use at 52 weeks has been reported for patients who underwent repeated series of bioengineered HA [17]. Chitnis and coworkers examined the utilization 
of nonsteroidal anti-inflammatory drugs (NSAIDs), CS injections, and opioids in the 6 months before and after HA treatment period, using commercial claims data [18]. In their cohort of 29,076 patients, the proportion of patients filling NSAID, CS injection, and opioid prescriptions decreased by 6, 28 and 4\%, respectively. Using the same commercial claims database, McIntyre et al. observed a 15, 57 and 6\% reduction in the number of patients filling NSAID, CS injection and opioid prescriptions, respectively, in the 6 months after 152,953 patients received a HA injection compared with the 6-month pretreatment period [19]. After stratifying their HA patients into those who did and did not have a TKA in the 6 months after the index HA injection, they further found that an $85 \%$ increase in proportion of TKA patients compared with a $15 \%$ decrease in proportion of non-TKA patients filling an opioid prescription. A reduction of oral medication taken for knee OA pain by 68, 68 and $57 \%$ at weeks 4 , 12 and 26 after high molecular weight HA treatment was also reported by Waddell et al. [20].

The reduction in pain medication usage following HA therapy may be explained by the various potential mechanisms of action for HA in providing clinical benefit in knee OA [32]. One such mechanism is the analgesic effect through interaction with HA receptors and/or free nerve endings within the joint tissue, by decreasing mechanical sensitivity of the stretch-activated ion channels. Although we are unable to provide causal inferences from our observational results, HA may help reduce opioid use in patients who go onto KA, which in turn reduces the risk of postoperative opioid use and associated suboptimal outcomes post-KA. For patients who may be considering KA, HA may help wean them from opioids prior to KA so as to reduce the potential risk of opioid dependency following KA. HA may also help to reduce the need for additional CS injections, particularly in light of questions regarding the effects of repeated CS injections on accelerating cartilage damage [33,34].

\section{Conclusion}

Excessive opioid use carries substantial risks, as well as the potential for misuse, dependency and opioid-related adverse events. For patients who undergo KA, opioid usage in both the pre- and post-operative periods is associated with poorer patient satisfaction and increased morbidity. Thus, it is important to consider alternative, nonsurgical treatment options that can effectively reduce pain and in turn, the need for opioids. This study clearly demonstrates that patients injected with HA experience a substantial reduction in opioid prescriptions and CS injections.

\section{Summary points}

- Pain management is an important component of nonsurgical treatment in symptomatic knee osteoarthritis (OA) and often includes the use of opioids, which are not without risks.

- About one-fifth of knee OA patients filled opioid prescriptions, along with about one-quarter who required at least one corticosteroid (CS) injection, within a year of diagnosis.

- Over half of the opioid users discontinued filling new prescriptions after receiving a hyaluronic acid (HA) injection.

- After receiving HA injections, new opioid users remained low with $14 \%$ of nonusers initiating use.

- Overall, $82 \%$ of HA patients did not use opioids within 6 months following the HA injection.

- About two-thirds of knee arthroplasty (KA) patients filled opioid prescriptions 3-6 months postsurgery, with the vast majority (about $75 \%$ ) of those as a new opioid user.

- The proportion of new opioid users was found to be greater for KA patients than HA patients, while the proportion of opioid users who discontinued use was greater for HA patients than KA patients.

- Most patients receiving HA (78\%) also did not require additional CS injections in the 6 months after their HA injection.

- With increasing concerns surrounding the risks of opioid addiction, consideration should be given to whether alternative OA therapies, such as CS and HA injections, are effective in reducing the opioid burden.

\section{Financial \& competing interests disclosure}

The institution of some of the authors (E Lau, SM Kurtz, KL Ong) has received funding from Ferring Pharmaceuticals, Inc., for this study. VD Kidd certifies that he has no commercial associations (e.g., consultancies, stock ownership, equity interest, patent/licensing arrangements, etc.) that might pose a conflict of interest in connection with the submitted article. G Malanga is a paid presenter or speaker for Cephalon, Endo Pharm and Forrest Labs. SH Dysart is a paid consultant, paid presenter or speaker for Ferring Pharmaceuticals. He is a paid consultant; paid presenter or speaker; received research support; received stock or stock options for Pacira Pharmaceuticals. F Niazi is an employee of Ferring Pharmaceuticals, Inc. KL Ong is an employee and shareholder of Exponent, a scientific and engineering consulting firm. Exponent has been paid fees for his consulting services on behalf of such companies and suppliers (Medtronic, Stryker Orthopaedics, Sanofi, Ferring Pharmaceuticals, Paradigm Spine, Pacira Phar- 
maceuticals, St. Jude Medical, Relievant Medsystems, International Society for the Advancement of Spine Surgery, SI-Technology, LLC, Zimmer Biomet, Joerns Healthcare, SpineFrontier, Ethicon, DJO, Ossur, Karl Storz Endoscopy-America, Rex Medical). E Lau is an employee of Exponent, a scientific and engineering consulting firm. Exponent has been paid fees for his consulting services on behalf of such companies and suppliers (Alcon Corp, Boston Scientific, Ferring Pharmaceuticals, Medtronic, Relievant Medsystems, Sanofi-Aventis, Stryker). SM Kurtz is an employee and shareholder of Exponent, a scientific and engineering consulting firm. Exponent has been paid fees for his consulting services on behalf of such companies and suppliers (Ferring Pharmaceuticals, Celanese, Ceramtec, Formae, Invibio, Simplify Medical, Stelkast, Kyocera Medical, Wright Medical Technology, Lima Corporate, Stryker, Zimmer Biomet). The authors have no other relevant affiliations or financial involvement with any organization or entity with a financial interest in or financial conflict with the subject matter or materials discussed in the manuscript apart from those disclosed.

No writing assistance was utilized in the production of this manuscript.

Ethical conduct of research

This study was based on publicly available data sets, did not use private health identifiable information and did not represent human subject research.

Open access

This work is licensed under the Attribution-NonCommercial-NoDerivatives 4.0 Unported License. To view a copy of this license, visit http://creativecommons.org/licenses/by-nc-nd/4.0/

\section{References}

Papers of special note have been highlighted as: $\bullet \bullet$ of considerable interest

1. Dowell DH, Tamara M, Chou R. CDC Guideline for Prescribing Opioids for Chronic Pain - United States, 2016. MMWR Recomm. Rep. 65(No. RR-1), 1-49 (2016).

2. Frieden TR, Houry D. Reducing the risks of relief - the cdc opioid-prescribing guideline. N. Engl. 374(16), 1501-1504 (2016).

- $\quad$ CDC published guidelines the appropriateness of prescribing opioids for chronic pain.

3. American Academy of Orthopaedic Surgeons. Treatment of Osteoarthritis of the Knee: Evidence-Based Guideline. (2nd Edition) (2013). www.aaos.org/research/guidelines/TreatmentofOsteoarthritisof theKneeGuideline.pdf

- Despite concerns regarding chronic opioid use, opioids have been given inconclusive recommendation for its use to manage knee osteoarthritispain by the American Academy of Orthopaedic Surgeons.

4. DeMik DE, Bedard NA, Dowdle SB, Burnett RA, McHugh MA, Callaghan JJ. Are we still prescribing opioids for osteoarthritis? J. Arthroplasty 32(12), 3578-3582 e3571 (2017).

5. Vina ER, Hausmann LRM, Obrosky DS et al. Social \& psychological factors associated with oral analgesic use in knee osteoarthritis management. Osteoarthr. Cartil. 27(7), 1018-1025 (2019).

6. Desai RJ, Jin Y, Franklin PD et al. Association of geography and access to healthcare providers with long term prescription opioid use in Medicare patients with severe osteoarthritis: a cohort study. Arthritis Rheumatol. 71(5), 712-721 (2019).

7. Bedard NA, Pugely AJ, Westermann RW, Duchman KR, Glass NA, Callaghan JJ. Opioid use after total knee arthroplasty: trends and risk factors for prolonged use. J. Arthroplasty 32(8), 2390-2394 (2017).

8. Inacio MCS, Cashman K, Pratt NL et al. Prevalence and changes in analgesic medication utilisation 1 year prior to total joint replacement in an older cohort of patients. Osteoarthr. Cartil. 26(3), 356-362 (2018).

9. Jain N, Brock JL, Malik AT, Phillips FM, Khan SN. Prediction of complications, readmission, and revision surgery based on duration of preoperative opioid use: analysis of major joint replacement and lumbar fusion. J. Bone Joint Surg. Am. 101(5), 384-391 (2019).

-• Pre-operative use of opioids is associated with greater risk of readmission, complications and revision surgery following total knee replacement surgery.

10. Kim SC, Choudhry N, Franklin JM et al. Patterns and predictors of persistent opioid use following hip or knee arthroplasty. Osteoarthr. Cartil. 25(9), 1399-1406 (2017).

11. Manalo JPM, Castillo T, Hennessy D, Peng Y, Schurko B, Kwon YM. Preoperative opioid medication use negatively affect health related quality of life after total knee arthroplasty. Knee 25(5), 946-951 (2018).

12. Namba RS, Singh A, Paxton EW, Inacio MCS. Patient factors associated with prolonged postoperative opioid use after total knee arthroplasty. J. Arthroplasty 33(8), 2449-2454 (2018).

13. Politzer CS, Kildow BJ, Goltz DE, Green CL, Bolognesi MP, Seyler TM. Trends in opioid utilization before and after total knee arthroplasty. J. Arthroplasty 33(7S), S147-S153 e141 (2018).

14. Smith SR, Bido J, Collins JE, Yang H, Katz JN, Losina E. Impact of preoperative opioid use on total knee arthroplasty outcomes. J. Bone Joint Surg. Am. 99(10), 803-808 (2017). 
15. Zarling BJ, Yokhana SS, Herzog DT, Markel DC. Preoperative and postoperative opiate use by the arthroplasty patient. J. Arthroplasty 31(10), 2081-2084 (2016).

-. Pre-operative opioid use before total knee replacement surgery is a significant risk factor for greater postoperative opioid use.

16. Namba RS, Inacio MCS, Pratt NL, Graves SE, Roughead EE, Paxton EW. Persistent opioid use following total knee arthroplasty: a signal for close surveillance. J. Arthroplasty 33(2), 331-336 (2018).

-• Persistent opioid use following knee replacement surgery may also have negative clinical consequences.

17. Altman RD, Rosen JE, Bloch DA, Hatoum HT. Safety and efficacy of retreatment with a bioengineered hyaluronate for painful osteoarthritis of the knee: results of the open-label Extension Study of the FLEXX Trial. Osteoarthr. Cartil. 19(10), 1169-1175 (2011).

-. Hyaluronic acid (HA) injections has been reported to reduce the need for analgesic or rescue medication, although HA injections were not recommended by the American Academy of Orthopaedic Surgeons for managing knee osteoarthritis pain.

18. Chitnis AS, Etter K, Holy CE, Gray FS, Fernando JM, Bisson B. Real world impact of the high concentration non-avian high molecular weight hyaluronan on pain medication use among osteoarthritis patients. Curr. Med. Res. Opin. 35(9), 1523-1527 (2019).

19. McIntyre LF, Beach W, Bhattacharyya S, Yadalam S, Bisson B, Kim M. Impact of hyaluronic acid injections on utilization of pain management medications. Am. J. Pharma Benefits 9(6), 195-199 (2017).

20. Waddell DD, Bricker DC. Clinical experience with the effectiveness and tolerability of hylan G-F 20 in 1047 patients with osteoarthritis of the knee. J. Knee Surg. 19(1), 19-27 (2006).

21. Manchikanti L, Kaye AM, Knezevic NN et al. Responsible, safe, and effective prescription of opioids for chronic non-cancer pain: American Society of Interventional Pain Physicians (ASIPP) Guidelines. Pain Physician 20(2S), S3-S92 (2017).

22. Ben-Ari A, Chansky H, Rozet I. Preoperative opioid use is associated with early revision after total knee arthroplasty: a study of male patients treated in the veterans affairs system. J. Bone Joint Surg. Am. 99(1), 1-9 (2017).

23. Cancienne JM, Patel KJ, Browne JA, Werner BC. Narcotic use and total knee arthroplasty. J. Arthroplasty 33(1), 113-118 (2018).

24. Alenazi AM, Obaidat SM, Alshehri MM, Alothman S, Gray C, Rucker J. Type 2 diabetes affects joint pain severity in people with localized osteoarthritis: a retrospective study. Pain Med. 21(5), 1025-1031 (2020).

25. Alenazi AM, Alshehri MM, Alothman S et al. The association of diabetes with knee pain locations, pain while walking, and walking speed: data from the osteoarthritis initiative. Phys. Ther. (2020) (Epub ahead of print).

26. Eitner A, Culvenor AG, Wirth W, Schaible HG, Eckstein F. Impact of diabetes mellitus on knee osteoarthritis pain and physical and mental status: data from the osteoarthritis initiative. Arthritis Care Res. (2020) (Epub ahead of print).

27. Han B, Compton WM, Blanco C, Crane E, Lee J, Jones CM. Prescription opioid use, misuse, and use disorders in U.S. adults: 2015 national survey on drug use and health. Ann. Intern. Med. 167(5), 293-301 (2017).

28. Rashidian H, Hadji M, Marzban M et al. Sensitivity of self-reported opioid use in case-control studies: healthy individuals versus hospitalized patients. PLoS One 12(8), e0183017 (2017).

29. Kessler ER, Shah M, Gruschkus SK, Raju A. Cost and quality implications of opioid-based postsurgical pain control using administrative claims data from a large health system: opioid-related adverse events and their impact on clinical and economic outcomes. Pharmacotherapy 33(4), 383-391 (2013).

30. Beswick AD, Wylde V, Gooberman-Hill R, Blom A, Dieppe P. What proportion of patients report long-term pain after total hip or knee replacement for osteoarthritis? A systematic review of prospective studies in unselected patients. BMJ Open 2(1), e000435 (2012).

31. Altman RD, Schemitsch E, Bedi A. Assessment of clinical practice guideline methodology for the treatment of knee osteoarthritis with intra-articular hyaluronic acid. Semin. Arthritis Rheu. 45(2), 132-139 (2015).

32. Altman RD, Manjoo A, Fierlinger A, Niazi F, Nicholls M. The mechanism of action for hyaluronic acid treatment in the osteoarthritic knee: a systematic review. BMC Musculoskelet. Disord. 16, 321 (2015).

-. Reduction in pain medication usage following intra-articular HA therapy may be explained by the various potential mechanisms of action for HA in providing clinical benefit in knee osteoarthritis.

33. McAlindon TE, LaValley MP, Harvey WF et al. Effect of intra-articular triamcinolone vs saline on knee cartilage volume and pain in patients with knee osteoarthritis: a randomized clinical trial. JAMA 317(19), 1967-1975 (2017).

34. Wernecke C, Braun HJ, Dragoo JL. The effect of intra-articular corticosteroids on articular cartilage: a systematic review. Orthop. J. Sports Med. 3(5), https://doi.org/10.1177/2325967115581163 (2015) (Epub ahead of print). 
